\title{
Contact problems between a functionally graded layer and a rigid support
}

\author{
M. Abanoz ${ }^{1}$, M. Yaylac1 ${ }^{1 *}$ and A. Birinci ${ }^{2}$ \\ ${ }^{1}$ Recep Tayyip Erdoğan University, Department of Civil Engineering, Rize, Turkey \\ ${ }^{2}$ Karadeniz Technical University, Department of Civil Engineering, Trabzon, Turkey
}

\begin{abstract}
In this study, frictionless contact problem for a functionally graded (FG) layer is considered. The FG layer is subjected to load with a rigid stamp and the FG layer is bonded on a rigid foundation. The graded layer is modeled as a non-homogenous medium with a constant Poisson's ratio and exponentially varying shear modules. It is assumed that the contact between all surfaces is frictionless and the effect of gravity force is neglected. The problem is solved analytically using plane elasticity and integral transform techniques. The problem is reduced to a singular integral equation using plane elasticity and integral transform techniques. Obtained singular integral equation is solved numerically using Gauss-Jacobi integration formulation and obtain the contact pressure and contact length. The contact length and contact pressures between the FG layer and the rigid stamp are analyzed for various material properties and loading. Aim of the paper is to investigate the effect of the non-homogeneity parameter of the graded layer on the contact pressures and lengths.
\end{abstract}

\section{Keywords}

Contact problem; Functionally graded layer; Singular integral equations; Gauss-Jacobi integration

Received: 19 January 2019; Accepted: 04 March 2019

ISSN: 2630-5763 (online) C 2019 Golden Light Publishing ${ }^{\circledR}$ All rights reserved.

\section{Introduction}

Materials with changing composition, microstructure, or porosity across the volume of the material are referred to as the functionally graded material (FGM) [1]. Functionally graded materials are inhomogeneous materials, consisting of different materials, performed to have a continually modify spatial composition profile [2]. Functionally graded materials are modern engineering materials designed for a specific performance or function in which a spatial gradation in structure [3]. The major property of the FGM have made them to be advantageous in almost all the human areas of profession. Functionally graded materials are now being applied in a number of industries, with a big potential to be used in other practices in the future. The current applications are aerospace, automobile, biomedical, defense, electrical/electronic, energy, marine mechanical [4].

The axisymmetric problem of a functionally graded half-space forced to a concentrated load studied by Giannakopoulos and Suresh [5]. Guler and Erdogan investigated a series of analytical comparison results for the contact problem of two deformable solids containing a graded coating with exponentially varying shear modulus [6]. Receding contact problem between a functionally graded coating and a homogeneous substrate is examined by El-Borgi et al. [7]. Barik et al. concerned with

Corresponding author

E-mail: murat.yaylaci@erdogan.edu.tr 
the stationary plane contact of a functionally graded heat conducting punch and a rigid insulated halfspace [8]. The two-dimensional frictionless contact problem of a coating structure consisting of a surface coating, a functionally graded layer and a substrate under a rigid cylindrical punch is investigated by Yang and Ke [9]. Rhimi et al. investigated the effect of the material nonhomogeneity parameter and the thickness of the graded layer on the contact pressure and on the length for the axisymmetric problem of a frictionless receding contact between an elastic functionally graded layer and a homogeneous halfspace [10]. The axisymmetric problem of a frictionless double receding contact between a rigid stamp of axisymmetric profile, an elastic functionally graded layer and a homogeneous half space is investigated by Rhimi et al. [11]. Chen and Chen studied a rigid punch contacting with a graded layer on a rigid substrate that heat generated by contact friction is investigated with a constant friction coefficient and inertia effects are neglected [12]. Comez studied a contact problem for a functionally graded layer loaded by means of a rigid stamp and supported by a Winkler foundation [13]. Çömez also examined contact problem for a functionally graded layer indented by a moving punch [14]. The plane problem of a smooth double receding contact between a functionally graded layer and an elastic layer when they are pressed together is investigated by Yan and Li [15]. ElBorgi and Çömez studied the plane problem of a receding frictional nonlinear contact between an elastic graded layer and a homogeneous half-space that pressed against each other by a rigid stamp [16]. Güler et al. developed and computational methods for the plane frictional contact problem of a cylindrical punch on a functionally graded orthotropic medium [17]. Turan et. al. [18], Adiyaman et al. [19-20] and Oner et. al. [21] and studied the contact problem of a functionally graded layer. The buckling analysis of FGM circular truncated conical and cylindrical shells subjected to combined axial extension loads and hydrostatic pressure and resting on a Pasternak type elastic foundation is examined by Sofiyev [22]. The non-linear free vibration behavior of functionally graded orthotropic cylindrical shell interacting with the two-parameter elastic foundation is studied by Sofiyev et. al. [23].

\section{Formulation of the problem}

As shown in Fig. 1, consider the symmetric plane strain problem consists of an infinitely long functionally graded (FG) layer which bonded to a rigid support on its lower surface is investigated according to the theory of elasticity. The thickness of FG layer is $h$ and a concentrated force $P$ is to the layer via a rigid cylindrical punch with radius $R$. Poisson's ratio $v$ is taken as constant, the shear modulus $\mu$ depend on the $y$-coordinate only as follows:

$$
\mu(y)=\mu_{0} e^{\beta y} \quad(0 \leq y \leq h)
$$

where $\mu_{0}$ are the shear modules of the graded layer at $y=0, \beta$ are the non-homogeneity parameter controlling the variation of the shear modules in the graded layer. It is assumed that the contact surfaces are frictionless and $x=0$ is to be the plane of symmetry with respect to external loads as well as geometry, for simplicity. Clearly, it is sufficient to consider one half (i.e., $x \geq 0$ ) of the medium only.

Assuming that the FG layer is isotropic at every point, equilibrium equations, the straindisplacement relationships and the linear elastic stress-strain law, respectively, are given by:

$$
\begin{aligned}
& \frac{\partial \sigma_{x}}{\partial x}+\frac{\tau_{x y}}{\partial y}=0, \quad \frac{\partial \tau_{y x}}{\partial x}+\frac{\partial \sigma_{y}}{\partial y}=0 \\
& \varepsilon_{x x}=\frac{\partial u}{\partial x}, \quad \varepsilon_{y y}=\frac{\partial v}{\partial y}, \quad \varepsilon_{x y}=\frac{1}{2}\left(\frac{\partial u}{\partial y}+\frac{\partial v}{\partial x}\right) \\
& \sigma_{x}=\frac{\mu(y)}{\kappa-1}\left[(1+\kappa) \varepsilon_{x x}+(3-\kappa) \varepsilon_{y y}\right] \\
& \sigma_{y}=\frac{\mu(y)}{\kappa-1}\left[(3-\kappa) \varepsilon_{x x}+(1+\kappa) \varepsilon_{y y}\right] \\
& \tau_{x y}=2 \mu(y) \varepsilon_{x y}
\end{aligned}
$$



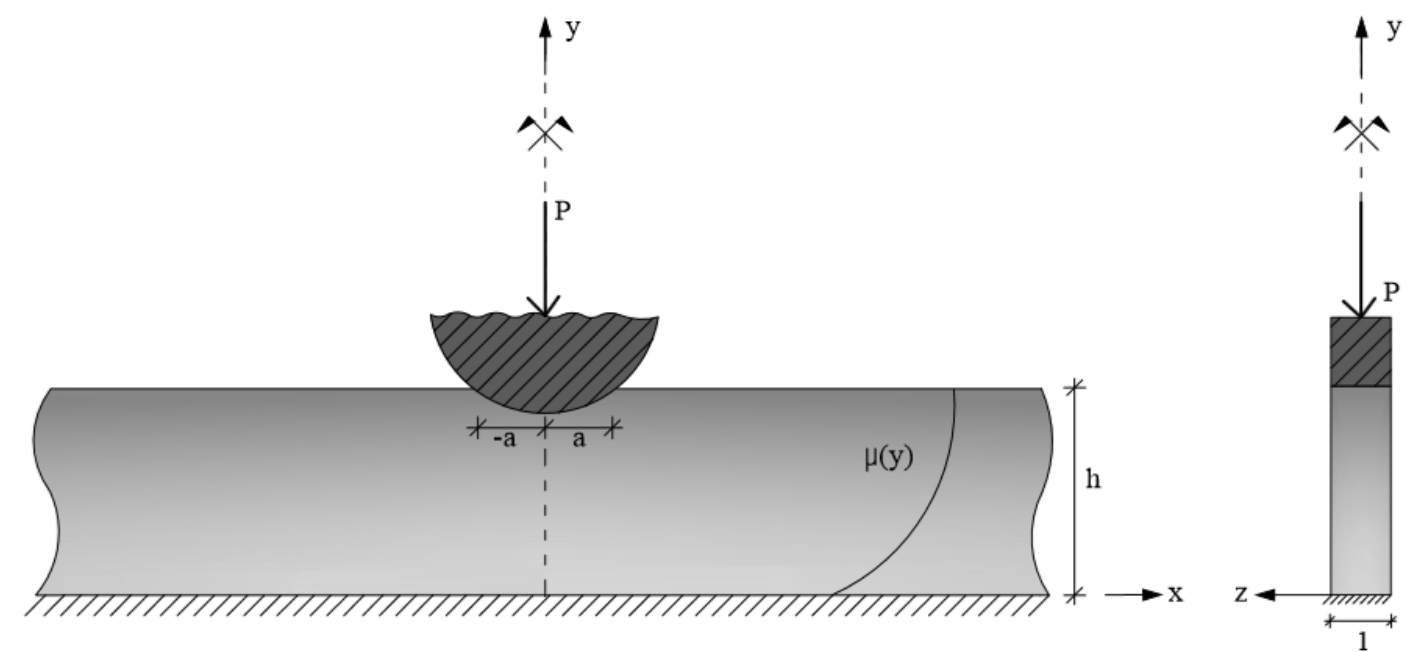

Fig. 1. Geometry and loading of the contact problem

where $u$ and $v$ are the $\mathrm{x}$ and $\mathrm{y}$ components of the displacement field, respectively; $\sigma_{x}, \sigma_{y}$ and $\tau_{x y}$ are the components of the stress field in the same coordinate system; $\varepsilon_{x}, \varepsilon_{y}$ and $\varepsilon_{x y}$ are the corresponding components of the strain field; $\kappa$ is a material property defined as $\kappa=3-4 v$ for plane strain problems. Combining Eqs. (1)-(4), the following two-dimensional Navier's equations are obtained:

$$
\begin{array}{r}
(\kappa+1) \frac{\partial^{2} u}{\partial x^{2}}+(\kappa-1) \frac{\partial^{2} u}{\partial y^{2}}+2 \frac{\partial^{2} v}{\partial x \partial y} \\
+\beta(\kappa-1) \frac{\partial u}{\partial y}+\beta(\kappa-1) \frac{\partial v}{\partial x}=0 \\
(\kappa-1) \frac{\partial^{2} v}{\partial x^{2}}+(\kappa+1) \frac{\partial^{2} v}{\partial y^{2}}+2 \frac{\partial^{2} u}{\partial x \partial y} \\
+\beta(3-\kappa) \frac{\partial u}{\partial x}+\beta(\kappa+1) \frac{\partial v}{\partial y}=0
\end{array}
$$

In case of graded layer solution, using symmetry considerations and Fourier transforms, the displacement components for FG layer may be written:

$$
\begin{aligned}
& u_{h}(x, y)=\frac{2}{\pi} \int_{0}^{\infty} \phi(\xi, y) \sin (\xi x) d \xi \\
& v_{h}(x, y)=\frac{2}{\pi} \int_{0}^{\infty} \psi(\xi, y) \cos (\xi x) d \xi
\end{aligned}
$$

where $\phi(\xi, y)$ and $\psi(\xi, y)$ are the inverse Fourier sine and Fourier cosine transforms of $u$ and $v$ with respect to the $x$-coordinate and the $y$-coordinate, respectively. Substituting Eqs. (6) into Navier equations (5), the following ordinary differential equations are obtained:

$$
\begin{array}{r}
-(\kappa+1) \xi^{2} \phi+(\kappa-1) \frac{d^{2} \phi}{d y^{2}}-2 \xi \frac{d \psi}{d y} \\
+\beta(\kappa-1)\left[\frac{d \phi}{d y}-\xi \psi\right]=0 \\
-(\kappa-1) \xi^{2} \psi+(\kappa+1) \frac{d^{2} \psi}{d y^{2}}+2 \xi \frac{d \phi}{d y} \\
+\beta\left[(3-\kappa) \xi \phi+(\kappa+1) \frac{d \psi}{d y}\right]=0
\end{array}
$$

where

$$
\phi=\sum_{j=1}^{4} A_{j} e^{n_{j} y}, \quad \psi=\sum_{j=1}^{4} A_{j} m_{j} e^{n_{j} y}
$$

The unknown functions $A_{j}(j=1,2,3,4)$ are determined from the boundary conditions and $n_{1}, \ldots, n_{4}$ are the four complex roots of the characteristic equation associated with Eqs. (7), which may be written:

$$
\begin{aligned}
n_{j}^{4}+2 \beta n_{j}^{3}+ & \left(\beta^{2}-2 \xi^{2}\right) n_{j}^{2}-2 \xi^{2} \beta n_{j} \\
& +\xi^{2}\left(\xi^{2}+\beta^{2} \frac{3-\kappa}{\kappa+1}\right)=0
\end{aligned}
$$


roots of which are:

$$
\begin{aligned}
& n_{1}=-\frac{1}{2}\left(\beta+\sqrt{4 \xi^{2}+\beta^{2}-4 \xi \beta i \sqrt{\frac{3-\kappa}{\kappa+1}}}\right), \\
& n_{2}=-\frac{1}{2}\left(\beta-\sqrt{4 \xi^{2}+\beta^{2}-4 \xi \beta i \sqrt{\frac{3-\kappa}{\kappa+1}}}\right), \\
& n_{3}=-\frac{1}{2}\left(\beta+\sqrt{4 \xi^{2}+\beta^{2}+4 \xi \beta i \sqrt{\frac{3-\kappa}{\kappa+1}}}\right), \\
& n_{4}=-\frac{1}{2}\left(\beta-\sqrt{4 \xi^{2}+\beta^{2}+4 \xi \beta i \sqrt{\frac{3-\kappa}{\kappa+1}}}\right)
\end{aligned}
$$

The known function $m_{j}$ in $2^{\text {nd }}$ expression in Eq. (8b) may be expressed as follows:

$$
m_{j}=\frac{\left(3 \beta+2 n_{j}-\beta \kappa\right)\left[n_{j}\left(\beta+n_{j}\right)(\kappa+1)-\xi^{2}(\kappa+3)\right]}{\xi\left[4 \xi^{2}-\beta^{2}(\kappa-3)(\kappa+1)\right]}
$$

Substituting Eqs. (6) and (8) into Eqs. (4), stress and displacement fields of interest for graded layer are obtained:

$\sigma_{y_{h}}=\frac{2 \mu_{0} e^{(\beta y)}}{\pi(\kappa-1)} \int_{0}^{\infty} \sum_{j=1}^{4} A_{j} C_{j} \mathrm{e}^{\left(n_{j} y\right)} \cos (\xi x) d \xi$

$$
\tau_{x y_{h}}=\frac{2 \mu_{0} \mathrm{e}^{(\beta y)}}{\pi} \int_{0}^{\infty} \sum_{j=1}^{4} A_{j} D_{j} e^{\left(n_{j} y\right)} \sin (\xi x) d \xi
$$

$v_{h}=\frac{2}{\pi} \int_{0}^{\infty} \sum_{j=1}^{4} A_{j} m_{j} \mathrm{e}^{\left(n_{j} y\right)} \cos (\xi x) d \xi$

$$
u_{h}=\frac{2}{\pi} \int_{0}^{\infty} \sum_{j=1}^{4} A_{j} e^{\left(n_{j} y\right)} \sin (\xi x) d \xi
$$

where $C_{j}$ and $D_{j}(j=1,2,3,4)$ are given by:

$$
\begin{aligned}
& C_{j}=(3-\kappa) \xi+(\kappa+1) m_{j} n_{j}, \\
& D_{j}=n_{j}-\xi m_{j}
\end{aligned}
$$

\section{Solution of the problems}

The boundary conditions of the contact problem for the FG layer can be written as:

$$
u(x, 0)=0, \quad(0 \leq x<\infty)
$$

$v(x, 0)=0, \quad(0 \leq x<\infty)$

$\tau_{x y}(x, h)=0, \quad(0 \leq x<\infty)$

$\sigma_{y}(x, h)=\left\{\begin{array}{cc}-p(x) & (0 \leq x<a) \\ 0 & (0 \leq x<\infty)\end{array}\right\}$

$\frac{\partial}{\partial x}[v(x, h)]=f(x), \quad(0 \leq x<a)$

where $p(x)$ is the unknown contact stress between the rigid punch and the layer on the contact area $(-a, a)$ and $f(x)$ is the derivative of the profile of the rigid stamp; one may write

$$
f(x)=\frac{x}{R}
$$

By making use of the boundary conditions given by (14a-d), the following matrix equation is obtained for the unknown constants $A_{j}(\mathrm{j}=1-4)$ appearing in the stress and displacement expressions for the FG layer in Eqs. (12):

$$
\left[\begin{array}{cccc}
D_{1} e^{\left(n_{1} h\right)} & D_{2} e^{\left(n_{2} h\right)} & D_{3} e^{\left(n_{3} h\right)} & D_{4} e^{\left(n_{4} h\right)} \\
C_{1} e^{\left(n_{1} h\right)} & C_{2} e^{\left(n_{1} h\right)} & C_{3} e^{\left(n_{1} h\right)} & C_{4} e^{\left(n_{1} h\right)} \\
m_{1} & m_{2} & m_{3} & m_{4} \\
1 & 1 & 1 & 1
\end{array}\right]\left\{\begin{array}{l}
A_{1} \\
A_{2} \\
A_{3} \\
A_{4}
\end{array}\right\}=\left\{\begin{array}{c}
0 \\
-P \\
0 \\
0
\end{array}\right\}
$$

where $P$ is defined as:

$P=\frac{\left(\kappa_{1}-1\right)}{\mu_{0} e^{\beta h}} \int_{0}^{\infty} p(x) \cos (\xi x) d x$

The unknown functions $A_{j}(\mathrm{j}=1-4)$ are obtained in the form

$$
A_{j}=\frac{A_{j 1}}{\Delta A} P
$$

where $A_{j 1}(j=1, \ldots, 4)$ and $\Delta A$ are given in Appendix.

Substituting Eqs. (19) into the remaining boundary condition (12) and using the symmetry consideration, $p(x)=p(-x)$, and after some routine manipulations 
$\frac{2}{\pi \mu_{0} e^{\beta h}} \int_{-a}^{a}\left[\frac{\kappa+1}{8}\left(\frac{1}{x-t}\right)+N(x, t)\right] p(t) d t=\frac{x}{R}$

where $N(x, t)$ is given in Appendix. In the singular integral equation (20), the contact area $a$ is also unknown, as well as the contact stress $p(x)$. For a complete solution of the problem, the contact stress $p(x)$ must satisfy the following equilibrium condition:

$$
\int_{-a}^{a} p(t) d t=P
$$

\section{The solution of the system of integral equations}

To simplify the numerical analysis of the integral equation, the following dimensionless quantities can be introduced.

$$
\begin{aligned}
& t=a r \\
& x=a s \\
& z=\xi h \\
& \phi(r)=\frac{h}{P} P(t)
\end{aligned}
$$

Using these dimensionless quantities, the integral Eqs. (20) and (21) can be written as

$$
\begin{aligned}
& \int_{-1}^{1}\left[\frac{\kappa+1}{8} \frac{1}{s-r}+\frac{a}{h} k(s, r)\right] \phi(r) d r \\
& =\frac{\pi e^{\beta h}}{2 R / h} \frac{\mu_{0}}{P / h} \frac{a}{h} s \\
& \frac{a}{h} \int_{-1}^{1} \phi(r) d r=1
\end{aligned}
$$

where $k(s, r)$ is given Appendix.

The solution of the integral equations can be expressed as

$$
\phi(r)=w(r) g(r)
$$

Since there are smooth contacts at the end points, the index of the integral equation (23a) is -1 [24].

$$
w(r)=(1-r)^{\alpha}(1+r)^{\beta}, \quad(\alpha=0.5, \beta=0.5)
$$

The solution of the integral equation can be expressed as Using the Gauss-Jacobi integration formulas, the integral Eq. (23a) and equilibrium conditions (23b) become,

$$
\begin{aligned}
& \sum_{i=1}^{N} W_{i}^{N} g\left(r_{i}\right)\left[\frac{\kappa+1}{8} \frac{1}{s_{k}-r_{i}}+\frac{a}{h} k\left(s_{k}, r_{i}\right)\right] \\
& =\frac{\pi e^{\beta h}}{2} \frac{\mu_{0}}{P / h} \frac{a / h}{R / h} s_{k} \quad k(1, \ldots, N+1) \\
& \frac{a}{h} \sum_{i=1}^{N} W_{i}\left(r_{i}\right) g\left(r_{i}\right)=1
\end{aligned}
$$

$r_{i}$ and $s_{k}$ are the roots of the related Jacobi polynomials and $W_{i}^{N}$ is the weighting constant given by

$$
\begin{aligned}
& r_{i}=\cos \left(\frac{i \pi}{N+1}\right) \quad(i=1, \ldots N) \\
& s_{k}=\cos \left(\frac{\pi}{2} \frac{2 k-1}{N+1}\right) \quad(k=1, \ldots N+1) \\
& W_{i}^{N}=\pi\left(\frac{1-r_{i}^{2}}{N+1}\right) \quad(i=1, \ldots N)
\end{aligned}
$$

Note that there are $N+1$ equations to determine the $N$ unknowns $\mathrm{g}\left(r_{i}\right)$ in Eq. (25a). Since the extra equation is used to normalise the interval of integration, it is sufficient to choose only $N$ of the $N+1$ possible collocation points [25]. Thus, Eqs. (25a) and (25b) give $N+1$ equations to determine the $N+1$ unknowns, which are $\mathrm{g}\left(r_{i}\right)$ and $a$. The system of equations is linear in terms of the $\mathrm{g}\left(r_{i}\right)$ but highly nonlinear in variable $a$. Therefore, an iterative method is used to obtain the unknowns.

\section{Numerical results}

This section presents numerical results for contact area and contact stress distribution of the FG layer due to frictionless contact of a rigid cylindrical punch. The values $\mu$ and $h$ should be considered fixed, they are related to more than one dimensionless quantity.

Table 1-2 and Figs. 2-3 show the variation of the contact width with inhomogeneity parameter $\beta$. $\beta h>0$ indicates that the rigidity of the top surface 
Table 1. Comparison of the contact widths $a / h$ under punch depending on load factor with $\beta(-1,0.001,1)$ and $\kappa=2$

\begin{tabular}{cccccccccc}
\hline \multirow{2}{*}{$\mu$} & \multicolumn{3}{c}{$R / h=10$} & \multicolumn{3}{c}{$R / h=100$} & \multicolumn{3}{c}{$R / h=1000$} \\
\cline { 2 - 10 } & -1 & 0.001 & 1 & -1 & 0.001 & 1 & -1 & 0.001 & 1 \\
\hline 100 & 0.3148 & 0.2152 & 0.1402 & 0.8203 & 0.6222 & 0.4574 & 1.9157 & 1.5453 & 1.2528 \\
200 & 0.2304 & 0.1533 & 0.0977 & 0.6233 & 0.4589 & 0.3235 & 1.4960 & 1.1906 & 0.9459 \\
400 & 0.1673 & 0.1088 & 0.0683 & 0.4680 & 0.3336 & 0.2262 & 1.1594 & 0.9083 & 0.7015 \\
500 & 0.1507 & 0.0974 & 0.0609 & 0.4257 & 0.3002 & 0.2013 & 1.0677 & 0.8303 & 0.6344 \\
800 & 0.1207 & 0.0771 & 0.0478 & 0.3473 & 0.2398 & 0.1575 & 0.8898 & 0.6839 & 0.5094 \\
1000 & 0.1085 & 0.0690 & 0.0427 & 0.3148 & 0.2152 & 0.1402 & 0.8203 & 0.6222 & 0.4574 \\
\hline
\end{tabular}

Table 2. Comparison of the contact widths $a / h$ under punch depending on the radius with $\beta(-1,0.001,1)$ and $\kappa=2$

\begin{tabular}{ccccccc}
\hline$R / h$ & \multicolumn{3}{c}{$\mu /(P / h)=100$} & & \multicolumn{3}{c}{$\mu /(P / h)=1000$} \\
\hline & -1 & 0.001 & 1 & -1 & 0.001 & 1 \\
10 & 0.3148 & 0.2152 & 0.1402 & 0.1085 & 0.0690 & 0.0427 \\
100 & 0.8203 & 0.6222 & 0.4574 & 0.3148 & 0.2152 & 0.1402 \\
500 & 1.4960 & 1.1906 & 0.9459 & 0.6233 & 0.4589 & 0.3235 \\
1000 & 1.9157 & 1.5453 & 1.2528 & 0.8203 & 0.6222 & 0.4574 \\
\hline
\end{tabular}
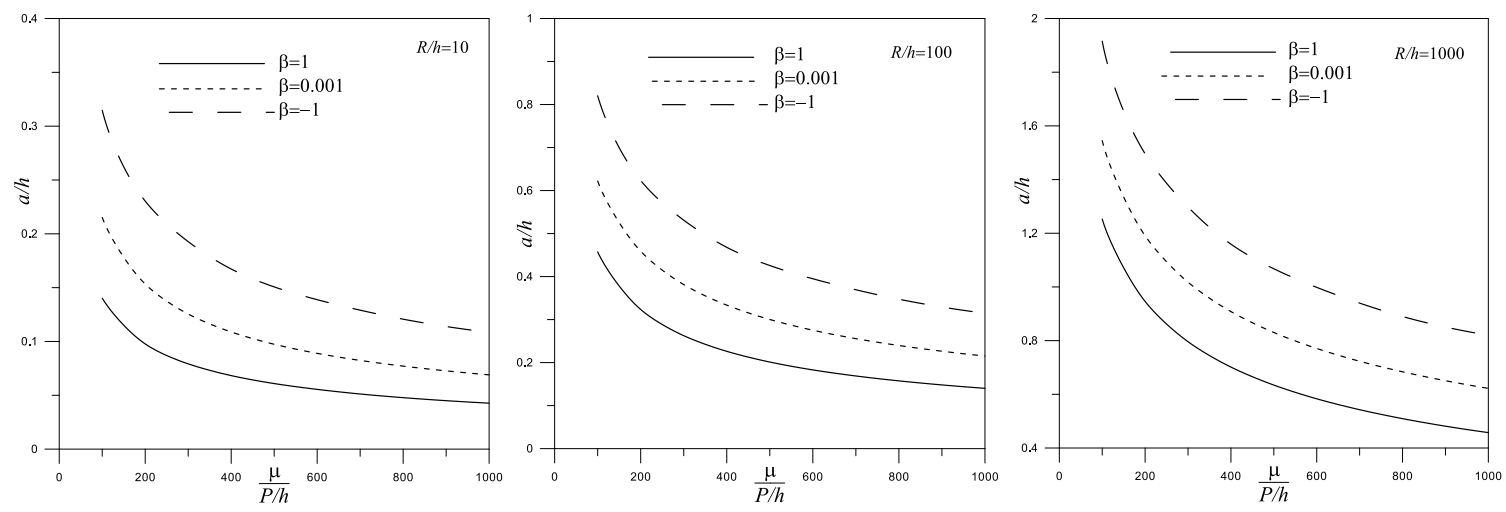

Fig. 2. Variation the contact area by depending on load factor $(\kappa=2)$

is higher than bottom surface of the layer. On the contrary when $\beta h<0$, rigidity is the opposite of the first case. When $\beta$ is getting increase, the contact width $a / h$ decrease. Tables 1 and 2 and Figs. 2 and 3 also show variation of the contact width by depending on the load factor $\mu /(P / h)$ and radius of punch $R / h$. Contact widths $a / h$ increases with increasing of radius of punch $R / h$. Since increasing load factor $\mu /(P / h)$ corresponds to decreasing the applied concentrated load $P$, the contact widths $a / h$ decreases.
In Table 3 and Fig. 4 the contact width $a / h$ are analyzed for inhomogeneity parameter $\beta$ by depending on the various of value quantities of the materials constant $\kappa$. With increasing inhomogeneity parameter $\beta$, the contact width $a / h$ decrease. In Table 3 and Fig. 4 are shown relation $a / h$ and $R / h$ by depending on material constant $\kappa$. Contact width $a / h$ increases with increasing material constant $\kappa$. Increasing of $\kappa$ corresponds to decreasing of Poisson ratio $v$. 
Figs. 5-8 illustrates the effect of the effect of the relative in inhomogeneity parameter on the contact pressure distribution on the top of the FG layer.

Figs. 5-7 show $P(x) /(P / h)$ the dimensionless contact pressure distributions. The contact pressure is maximum at $x=0$. In these figures, for $R / h=10$, 100 and 1000 , the relation among $\beta$ and $\mu /(P / h)$ is shown respectively. As load factor increases, dimensionless contact pressure increases. Also, with increasing contact stresses also increases when inhomogeneity parameter $\beta$ increases.
Fig. 8, shows $P(x) /(P / h)$ the dimensionless contact pressure distribution. In the figure, $\mu /(P / h)$ $=100$ and $\kappa=2$ are fixed and dimensionless contact pressure distribution for $R / h=10,100$ and 1000, is given. As $R / h$ increases, size of the contact area increases, so the applied load distributes a much larger area. Therefore, contact pressure decreases. With increasing inhomogeneity parameter $\beta$, the contact stresses decreasing.
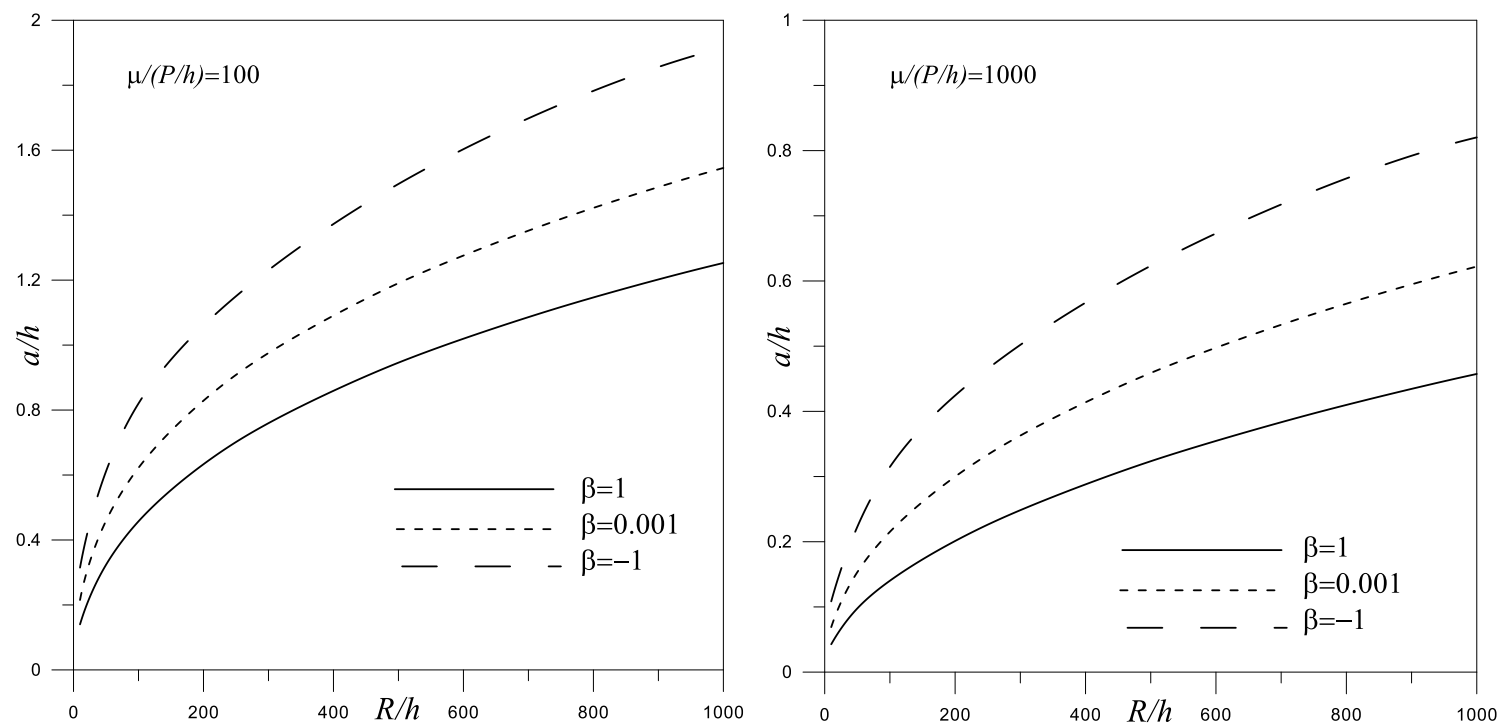

Fig. 3. Variation of the contact area by depending on the radius $(\kappa=2)$

Table 3. Comparison of the contact widths $a / h$ under punch depending on $\kappa$ with $\beta(-1,0.001,1)$ and $\mu /(P / h)=100$

\begin{tabular}{cccccccccc}
\hline$R / h$ & \multicolumn{3}{c}{$\kappa=1.5$} & \multicolumn{3}{c}{$\kappa=2.0$} & \multicolumn{3}{c}{$\kappa=2.5$} \\
\hline & -1 & 0.001 & 1 & -1 & 0.001 & 1 & -1 & 0.001 & 1 \\
10 & 0.2868 & 0.1965 & 0.1281 & 0.3148 & 0.2152 & 0.1402 & 0.3400 & 0.2323 & 0.1512 \\
50 & 0.5665 & 0.4190 & 0.2962 & 0.6233 & 0.4589 & 0.3235 & 0.6731 & 0.4946 & 0.3482 \\
100 & 0.7438 & 0.5676 & 0.4189 & 0.8203 & 0.6222 & 0.4574 & 0.8823 & 0.6702 & 0.4917 \\
250 & 1.0459 & 0.8259 & 0.6416 & 1.1594 & 0.9083 & 0.7015 & 1.2534 & 0.9787 & 0.7534 \\
500 & 1.3417 & 1.0779 & 0.8630 & 1.4960 & 1.1906 & 0.9459 & 1.6191 & 1.2839 & 1.0159 \\
750 & 1.5461 & 1.2528 & 1.0148 & 1.7419 & 1.3882 & 1.1172 & 1.8699 & 1.4979 & 1.2012 \\
1000 & 1.7125 & 1.3911 & 1.1369 & 1.9157 & 1.5453 & 1.2528 & 2.0781 & 1.6682 & 1.3471 \\
\hline
\end{tabular}



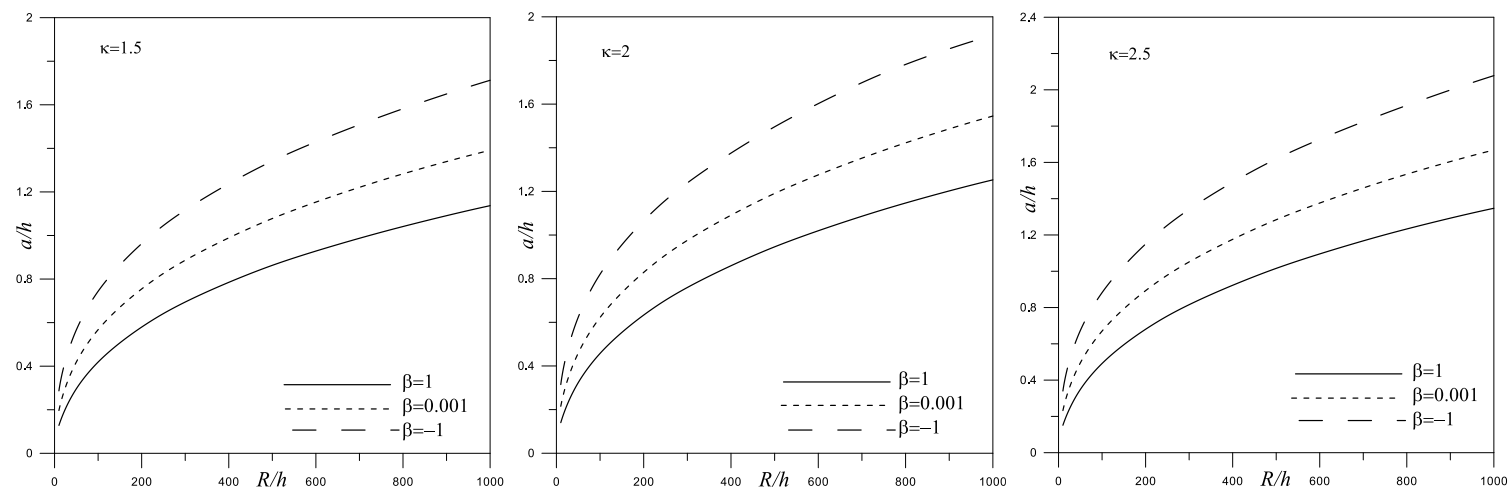

Fig. 4. Variation of the contact area by depending on $\kappa$ with $\mu /(P / h)=100$
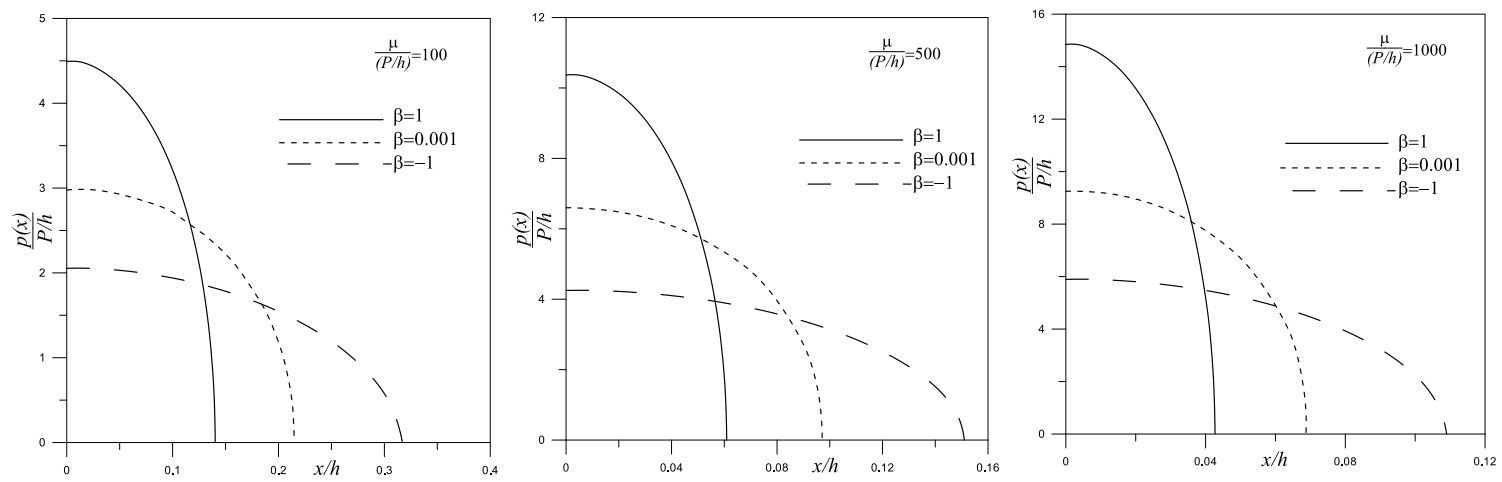

Fig. 5. The Contact pressure distribution with variation of load factor $(R / h=10, \kappa=2)$
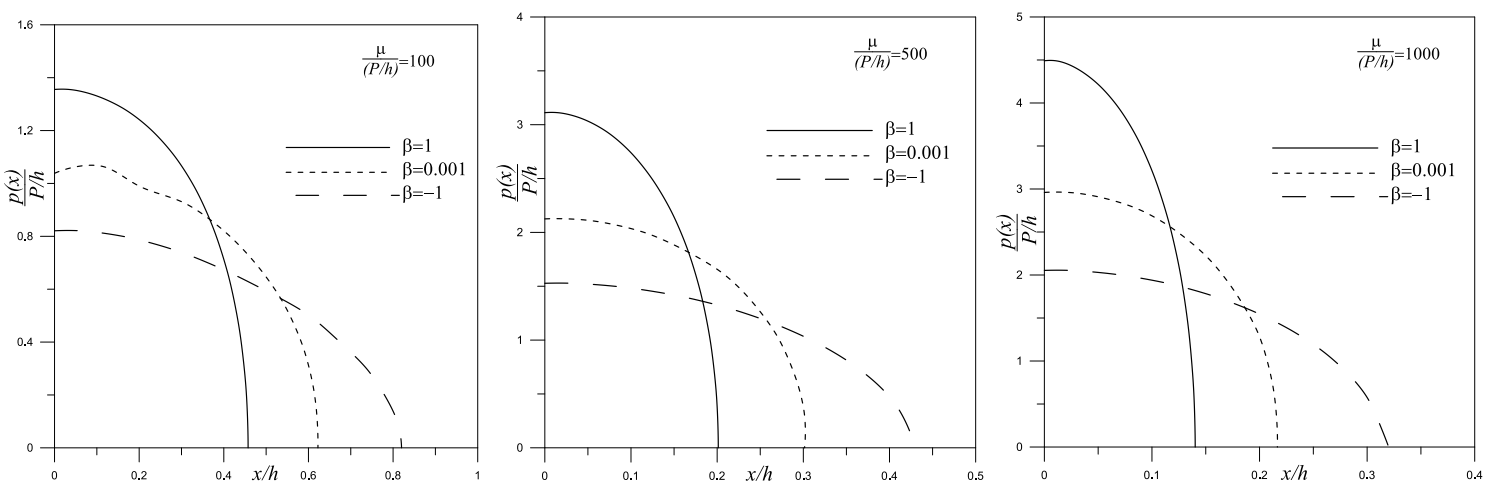

Fig. 6. The Contact pressure distribution with variation of load factor $(R / h=100, \kappa=2)$ 

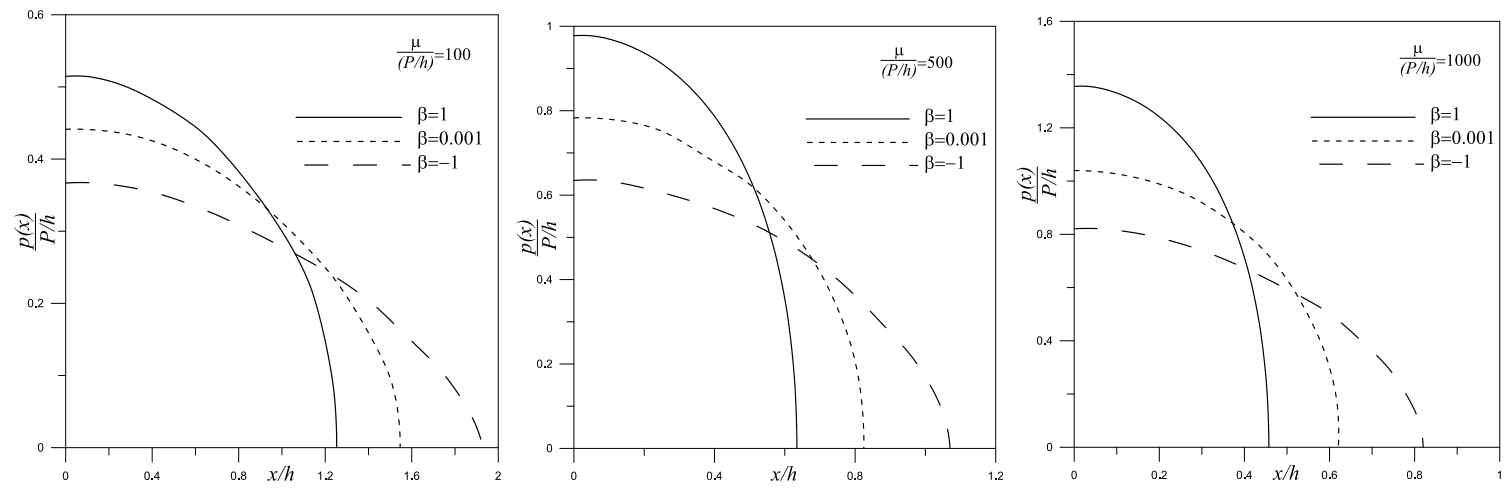

Fig. 7. The Contact pressure distribution with variation of load factor $(R / h=100, \kappa=2)$
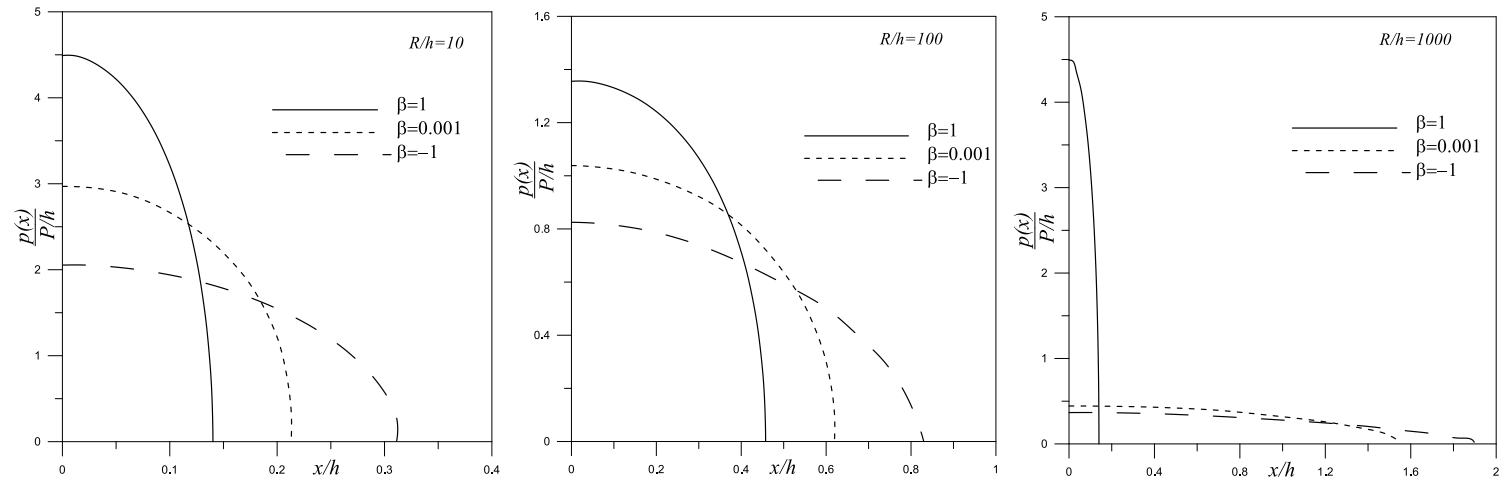

Fig. 8. The Contact pressure distribution with variation of radius $(\mu /(P / h)=100, \kappa=2)$

\section{Conclusion}

In this paper, the contact problem of a functionally graded (FG) layer resting on a rigid foundation is considered. The top of the FG layer is subjected to rigid cylindrical punch. The elasticity modulus is assumed to be exponential function. The problem is solved analytically using plane elasticity and integral transform techniques. The material inhomogeneity parameter has an important effect on the contact width and the stress distribution. The contact width $a / h$ and the stress distribution $P(x) /(P / h)$ are investigated for various material properties and loading, such as $\mu /(P / h), R / h$ and $\kappa$. The contact width $a / h$ decreases with increasing material inhomogeneity parameter $\beta$. The contact stress $P(x) /(P / h)$ increases with increasing material inhomogeneity parameter $\beta$. In addition, the greatest contact pressures occur at symmetry axis and increases with increasing $\beta$. With increasing load factor $\mu /(P / h)$ and radius of punch $R / h$ and material constant $\kappa$, the contact width $a / h$ increases. With increasing load factor $\mu /(P / h)$, radius of punch $R / h$ and material constant $\kappa$, the contact width $a / h$ increases. As it can be seen in the figures that increasing load factor $\mu /(P / h)$, contact pressure increases, but on the contrary contact pressure decreases with increasing radius of punch $R / h$.

\section{References}

[1] Nino M, Hirai T, Watanabe R (1987) The functionally gradient materials. Journal of the Japan Society for Composite Materials 13: 257264.

[2] Uduba G, Rao SS, Gangadharan KV (2014) Functionally graded composite materials: an overview. Procedia Material Science 5:1291-1299.

[3] Kawasaki A, Watanabe R (1997) Concept and P/M fabrication of functionally gradient materials. Ceramics International 23(1): 73-83. 
[4] Mahamood RM, Akinlabi ET. Functionally Graded Materials. Metallurgy and Materials Engineering. Springer International Publishing, 2017, DOI 10.1007/978-3-319-53756-6_2.

[5] Giannakopoulos AE, Suresh S (1997) Indentation of solids with gradients in elastic properties: Part I. Point force solution. International Journal of Solids and Structures 34: 2357-2392.

[6] Guler MA, Erdogan F (2006) Contact mechanics of two deformable elastic solids with graded coatings. Mechanics of Materials 38: 633-647.

[7] El-Borgi S, Abdelmoula R, Keer L (2006) A receding contact problem between a functionally graded layer and a homogeneous substrate. International Journal of Solids and Structures 43: 658-674.

[8] Barik SP, Kanoria M, Chaudhuri PK (2008) Steady state thermoelastic contact problem in a functionally graded material. International Journal of Engineering Science 46: 775-789.

[9] Yang J, Ke LL (2008) Two-dimensional contact problem for a coating-graded layer-substrate structure under a rigid cylindrical punch. International Journal of Mechanical Sciences 50: 985-994.

[10] Rhimi M, El-Borgi S, Ben Saïd W, Jemaa F (2009) A receding contact axisymmetric problem between a functionally graded layer and a homogeneous substrate. International Journal of Solids and Structures 46: 3633-3642.

[11] Rhimi M, El-Borgi S, Lajnef N (2011) A double receding contact axisymmetric problem between a functionally graded layer and a homogeneous substrate. Mechanics of Materials 43: 787-798.

[12] Chen P, Chen S (2012) Thermo-mechanical contact behavior of a finite graded layer under a sliding punch with heat generation. International Journal of Solids and Structures 50: 1108-1119.

[13] Comez I (2013) Contact problem of a functionally graded layer resting on a Winkler foundation. Acta Mechanica 224(11): 2833-2843.

[14] Comez I (2015) Contact problem for a functionally graded layer indented by a moving punch. International Journal Mechanical Science 100: 339-344.

[15] Yan J, Li X (2015) Double receding contact plane problem between a functionally graded layer and an elastic layer. European Journal of Mechanics A/Solids 53: 143-150.

[16] El-Borgi S, Çömez İ (2017) Receding frictional contact problem between a graded layer and a homogeneous substrate pressed by a rigid punch. Mechanics of Materials 114: 201-214.

[17] Güler MA, Kucuksucu A, Yilmaz KB, Yildirim B (2017) On the analytical and finite element solution of plane contact problem of a rigid cylindrical punch sliding over a functionally graded orthotropic medium. International Journal of Mechanical Sciences 120: 12-29.

[18] Turan M, Adiyaman G, Kahya V, Birinci A (2016) Axisymmetric analysis of a functionally graded layer resting on elastic substrate. Structural Engineering and Mechanics 58(3): 423-442.

[19] Adiyaman G, Birinci A, Öner E, Yaylacı M (2016) A receding contact problem between a functionally graded layer and two homogeneous quarter planes. Acta Mechanica 227(6): 1753-1766.

[20] Adiyaman G, Oner E, Birinci A (2017) Continuous and discontinuous contact problem of a functionally graded layer resting on a rigid foundation. Acta Mechanica 228(9): 303-317.

[21] Oner E, Adiyaman G, Birinci A (2017) Continuous contact problem of a functionally graded layer resting on an elastic half-plane. Archives of Mechanics 69(1): 53-73.

[22] Sofiyev AH (2010) Buckling analysis of FGM circular shells under combined loads and resting on the Pasternak type elastic foundation. Mechanics Research Communications 37: 539-544.

[23] Sofiyev AH, Hui D, Haciyev VC, Erdem H, Yuan GQ, Schnack E, Guldal V (2017) The nonlinear vibration of orthotropic functionally graded cylindrical shells surrounded by an elastic foundation within first order shear deformation theory. Composites Part B 116: 170-185.

[24] Erdogan F. Mixed boundary value problems in mechanics. In: Nemat-Nasser, S. Mechanics Today, vol. 4, Pergamon Press, New York, 1978.

[25] Krenk S (1975) On quadrature formulas for singular integral equations of the first and the second kind. Quarterly of Applied Mathematics 33: 225-232. 


\section{Appendix}

$$
\begin{aligned}
& A_{1}=-\left(P\left(D_{2} m_{3} e^{h n_{2}}-D_{2} m_{4} e^{h n_{2}}-D_{3} m_{2} e^{h n 3}+D_{3} m_{4} e^{h n_{3}}+D_{4} m_{2} e^{h n_{4}}-D_{4} m_{3} e^{h n_{4}}\right)\right) / \Delta \\
& A_{2}=\left(P\left(D_{1} m_{3} e^{h n_{1}}-D_{1} m_{4} e^{h n_{1}}-D_{3} m_{1} e^{h n 3}+D_{4} m_{1} e^{h n_{4}}+D_{3} m_{4} e^{h n_{3}}-D_{4} m_{3} e^{h n_{4}}\right)\right) / \Delta \\
& A_{3}=-\left(P\left(D_{1} m_{2} e^{h n_{1}}-D_{2} m_{1} e^{h n_{2}}-D_{1} m_{4} e^{h n_{1}}+D_{2} m_{4} e^{h n_{2}}+D_{4} m_{1} e^{h n_{4}}-D_{4} m_{2} e^{h n_{4}}\right)\right) / \Delta \\
& A_{4}=\left(P\left(D_{1} m_{2} e^{h n_{1}}-D_{1} m_{3} e^{h n_{1}}-D_{2} m_{1} e^{h n_{2}}+D_{2} m_{3} e^{h n_{2}}+D_{3} m_{1} e^{h n_{3}}-D_{3} m_{2} e^{h n_{3}}\right)\right) / \Delta \\
& \Delta A=\left(e^{h n_{1}} e^{h n_{2}}\left(C_{1} D_{2} m_{3}-C_{2} D_{1} m_{3}-C_{1} D_{2} m_{4}+C_{2} D_{1} m_{4}\right)+e^{h n_{1}} e^{h n_{3}}\left(-C_{1} D_{3} m_{2}+C_{3} D_{1} m_{2}+C_{1} D_{3} m_{4}-C_{3} D_{1} m_{4}\right)\right. \\
& +e^{h n_{1}} e^{h n_{4}}\left(C_{1} D_{4} m_{2}-C_{4} D_{1} m_{2}-C_{1} D_{4} m_{3}+C_{4} D_{1} m_{3}\right)+e^{h n_{2}} e^{h n_{3}}\left(C_{2} D_{3} m_{1}-C_{3} D_{2} m_{1}-C_{2} D_{3} m_{4}+C_{3} D_{2} m_{4}\right) \\
& \left.+e^{h n_{2}} e^{h n_{4}}\left(-C_{2} D_{4} m_{1}+C_{4} D_{2} m_{1}+C_{2} D_{4} m_{3}-C_{4} D_{2} m_{3}\right)+e^{h n_{3}} e^{h n_{4}}\left(C_{3} D_{4} m_{1}-C_{4} D_{3} m_{1}-C_{3} D_{4} m_{2}+C_{4} D_{3} m_{2}\right)\right) \\
& N(x, t)=\int_{0}^{\infty} \frac{\xi(\kappa-1)}{2 \Delta A}\left\{\left[e^{\left(n_{1}+n_{2}\right) h}\left(m_{1} m_{4} D_{2}-m_{1} m_{3} D_{2}+m_{2} m_{3} D_{1}-m_{2} m_{4} D_{1}\right)\right.\right. \\
& +e^{\left(n_{1}+n_{3}\right) h}\left(m_{1} m_{2} D_{3}-m_{1} m_{4} D_{3}-m_{2} m_{3} D_{1}+m_{3} m_{4} D_{1}\right) \\
& +e^{\left(n_{1}+n_{4}\right) h}\left(-m_{1} m_{2} D_{4}+m_{1} m_{3} D_{4}+m_{2} m_{4} D_{1}-m_{3} m_{4} D_{1}\right) \\
& +e^{\left(n_{2}+n_{3}\right) h}\left(-m_{1} m_{2} D_{3}+m_{2} m_{4} D_{3}+m_{1} m_{3} D_{2}-m_{3} m_{4} D_{2}\right) \\
& +e^{\left(n_{2}+n_{4}\right) h}\left(m_{1} m_{2} D_{4}-m_{2} m_{3} D_{4}-m_{1} m_{4} D_{2}+m_{3} m_{4} D_{2}\right) \\
& \left.\left.+e^{\left(n_{3}+n_{4}\right) h}\left(-m_{1} m_{3} D_{4}+m_{2} m_{3} D_{4}+m_{1} m_{4} D_{3}-m_{2} m_{4} D_{3}\right)+\frac{\kappa+1}{8}\right]\right\} \sin \xi(t-x) d \xi \\
& k(s, r)=\int_{0}^{\infty} \frac{\frac{z}{h}(\kappa-1)}{2 \Delta A}\left\{\left[e^{\left(n_{1}+n_{2}\right) h}\left(m_{1} m_{4} D_{2}-m_{1} m_{3} D_{2}+m_{2} m_{3} D_{1}-m_{2} m_{4} D_{1}\right)\right.\right. \\
& +e^{\left(n_{1}+n_{3}\right) h}\left(m_{1} m_{2} D_{3}-m_{1} m_{4} D_{3}-m_{2} m_{3} D_{1}+m_{3} m_{4} D_{1}\right) \\
& +e^{\left(n_{1}+n_{4}\right) h}\left(-m_{1} m_{2} D_{4}+m_{1} m_{3} D_{4}+m_{2} m_{4} D_{1}-m_{3} m_{4} D_{1}\right) \\
& +e^{\left(n_{2}+n_{3}\right) h}\left(-m_{1} m_{2} D_{3}+m_{2} m_{4} D_{3}+m_{1} m_{3} D_{2}-m_{3} m_{4} D_{2}\right) \\
& +e^{\left(n_{2}+n_{4}\right) h}\left(m_{1} m_{2} D_{4}-m_{2} m_{3} D_{4}-m_{1} m_{4} D_{2}+m_{3} m_{4} D_{2}\right) \\
& \left.\left.+e^{\left(n_{3}+n_{4}\right) h}\left(-m_{1} m_{3} D_{4}+m_{2} m_{3} D_{4}+m_{1} m_{4} D_{3}-m_{2} m_{4} D_{3}\right)+\frac{\kappa+1}{8}\right]\right\} \sin z\left(\frac{a r}{h}-\frac{a s}{h}\right) d z
\end{aligned}
$$

